\title{
PERCEPCIÓN Y PRÁCTICAS DE LA CULTURA KRISHNA DESDE UNA PERSPECTIVA DE CUIDADO PALIATIVO EN ENFERMERÍA
}

\author{
Sandra Patricia Carreño Corredor ${ }^{1}$ \\ David Stiven Tovar Ramírez ${ }^{2}$
}

\section{RESUMEN}

El objetivo del estudio fue describir la percepción y las prácticas de la cultura Krishna desde una perspectiva de cuidado paliativo, en la ciudad de Bogotá. Se desarrolló a partir de una metodología cualitativa, con entrevistas semiestructuradas a tres personas profesantes de esta comunidad religiosa durante el mes de octubre de 2018. Los resultados arrojaron cinco categorías temáticas: Percepción de vida es nacer de nuevo; el cuidado, un estilo de vida espiritual para el cuerpo y el alma; las prácticas que permiten una conexión con el Krishna; la enfermedad y la muerte, hay un nuevo renacimiento; la muerte como una puerta de entrada a la nueva de vida y de conexión con dios. Se concluye que es necesario que el personal de salud adquiera un cuidado culturalmente competente, en el que reconozca las cosmovisiones de los sujetos de cuidado frente a la vida, la muerte, la salud o la enfermedad y frente al proceso mismo de los cuidados paliativos.

Palabras Clave: Cuidados Paliativos, Cultura, Religión, Espiritualidad, Atención de Enfermería (DeCS)

\section{PERCEPTION AND PRACTICES OF KRISHNA CULTURE FROM A PALLIATIVE CARE PERSPECTIVE IN NURSING}

\begin{abstract}
The aim of the study was to describe the perception and practices of the Krishna culture from a palliative care perspective, in the city of Bogotá. It was developed from a qualitative methodology, with semi-structured interviews with three professors from this religious community during the month of October 2018. The results yielded five thematic categories: Perception of life is to be born again; care, a spiritual lifestyle for body and soul; practices that allow a connection with Krishna; sickness and death, there is a new rebirth. It is concluded that it is necessary for health personnel to acquire culturally competent care, in which they recognize the cosmovisions of the subjects of care in the face of life, death, health or illness and in the face of the palliative care process itself.
\end{abstract}

Key Words: Paliative Care, Culture, Religion, Spirituality, Nursing Care (DeCS)

Recibido 17-03-2019. Aceptado 25-05- 2019

DOI: https://doi.org/10.26752/revistaparadigmassh.v1i1.453

\section{INTRODUCCIÓN}

El cuidado cultural es necesario para ofrecer una atención holística y humanizada, una necesidad de gran relevancia debido a los procesos migratorios a nivel mundial y nacional, donde se buscan estrategias de inversión motivada por la expectativa de mayor ingreso y mejor calidad de vida en un futuro (Arteaga \& Ibáñez, 2018). Lo anterior, es relevante

\footnotetext{
${ }^{1}$ Enfermera, Instituto Nacional de Cancerología E.S.E. Estudiante de Maestría en Enfermería en Cuidados Paliativos, Universidad El Bosque. Bogotá, Colombia. Correo electrónico: spcarreno@unbosque.edu.co. orcid.org/0000-0002-4816-7921.

2Enfermero, Clínica Meta. Estudiante de Maestría en Enfermería en Cuidados Paliativos, Universidad El Bosque. Bogotá, Colombia. Correo electrónico: dtovarr@ unbosque.edu.co https://orcid.org/0000-0003-1844-9984/print
} 
en la atención de enfermería si se tiene en cuenta que el cuidado debe adaptarse a las necesidades individualizadas de las personas y considerar sus preferencias y creencias.

La Constitución Política de Colombia de 1991, muestra aspectos relevantes como la libertad de culto, que implica que todas las personas tienen libertad de expresar, vivir y manifestar su respectiva de fe/ cultura. Este aspecto también aparece en la Ley 1438 de 2011 y la Ley 1751 de 2015 de Colombia, que incluye un enfoque diferencial y de gestión de riesgo para reconocer y organizar el cuidado en salud de acuerdo con las particulares, vulnerabilidades y potencialidades de las personas y colectivos, teniendo en cuenta el carácter multiétnico y pluricultural en donde se valora, reconoce y respeta la diversidad en el país.

En el desarrollo epistemológico de enfermería en primer lugar el cuidado por el papel por la cultura en los cuidados enfocándose en la enfermería transcultural de Leininger, donde el profesional de enfermería reconoce y valora la importancia de los factores culturales y transculturales, adquiriendo un rol fundamental en el cuidado, estableciendo una la relación de cultura y cuidado, viendo al paciente en una forma única y transcendental pudiendo conocer la percepción y las prácticas de las diferentes culturas sobre el significado de cuidados culturales, valores, creencias y estilos de vida (Carrasquilla Baza \& Pérez Quintero, 2018; McFarland, Mixer, WebheAlamah, \& Burk, 2012).

Según la Organización Mundial de Salud (OMS), los cuidados paliativos implican "un enfoque que mejora la calidad de vida de los pacientes y sus familias que enfrentan el problema asociado con enfermedades que ponen en peligro la vida, a través de la prevención y el alivio del sufrimiento mediante la identificación temprana y la evaluación y tratamiento impecables del dolor y otros problemas físicos, psicosocial y espiritual". Esta definición en la actualidad, se ha ido transformando en la medida en que se avanza hacia cuidados que se ofrecen a lo largo de la trayectoria de la enfermedad y desde el diagnóstico mismo. Teniendo en cuenta lo anterior, los cuidados paliativos provistos por enfermería, no pueden desligar las creencias culturales y religiosas de las personas frente a temas tan importantes como la vida o la muerte, la salud o la enfermedad, entre otras; que son claras durante las fases terminales de la enfermedad.

Donde el aporte de conocimiento sobre la cultura o el movimiento hare krishna nace en Nueva York en 1966 también conocida cultura Vaisnava por un monje indio A. C. Bhaktivedanta Swami Prabhupada, como un nuevo movimiento religioso basado en el hinduismo, expandiéndose en Europa del Este e India, logrando abarcar en América Latina entre estos Colombia. Considerando su Dios Krisna "suprema personalidad de Dios". En Colombia se empieza a conocer el movimiento hace aproximadamente 36 años, contando con templos en 20 ciudades. Teniendo un alto número de profesantes a la cultura, colombianos buscando su identidad y quien es Dios, convirtiéndola en un estilo de vida (Ketola, 2004; Press, 2016a).

Dentro de las afirmaciones teológicas de sus profesantes se encuentra que "las personas son almas (atma), entidades no materiales, de naturaleza espiritual. Las almas no pertenecen a este universo material, sino a un universo espiritual perfecto, donde la vida es eterna, feliz y llena de conocimiento (todo lo contrario de este mundo material, donde existen la 
muerte, el sufrimiento y la ignorancia)" (...) La filosofía vaisnava lo define "En este universo, el alma está cubierta por cuerpo material, y este es la causa del sufrimiento", creen que el alma, en su estado original, posee un cuerpo espiritual, con forma humana.

Teniendo en cuenta la importancia de incluir cuidados paliativos culturalmente competentes en la práctica de enfermería, adaptados e individualizados para cada sujeto de cuidado, este artículo pretende hacer un acercamiento hacia la comprender la percepción y las prácticas de practicantes a la cultura religiosa Krishna en la ciudad de Bogotá, hacia los cuidados paliativos.

\section{METODOLOGÍA}

Este estudio se desarrolló a partir de una metodología cualitativa, con entrevistas semiestructuradas a tres personas profesantes de la comunidad religiosa krishna durante el mes de octubre de 2018 en la ciudad de Bogotá, templo Gournitay. Los tres participantes aceptaron participar voluntariamente y firmaron el consentimiento informado. Para la obtención de la información se realizaron visitas de acercamiento con los líderes, con el fin de que autorizaran el ingreso, recorrido en el templo y entrevista. Estas últimas fueron grabadas en cada uno de los casos, transcritas y sometidas a análisis de contenido.

Es importante manifestar que esta investigación fue considerada según la Resolución 8430 como sin riesgo y que fue considerado el respetó, dignidad, sentimientos, pensamientos, emociones e ideas de los participantes; así mismo se guardó la confidencialidad de su identidad.

\section{RESULTADOS}

Los resultados obtenidos se muestran en cinco categorías temáticas: Percepción de vida es nacer de nuevo; el cuidado, un estilo de vida espiritual para el cuerpo y el alma; las prácticas que permiten una conexión con el Krishna; la enfermedad y la muerte, hay un nuevo renacimiento; la muerte como una puerta de entrada a la nueva de vida y de conexión con dios; las cuales se describen a continuación.

\section{Percepción de vida es nacer de nuevo}

La vida es un estado orgánico de la fuerza interna de los seres humanos, animales, plantas, con una capacidad de adaptarse a cambios constantes que se producen en el medio donde se encuentran. La espiritualidad esta enlazada con la vida, como guía de comportamiento de las personas en relación con la vivencia de su enfermedad y la toma de decisiones en salud, tanto en los cuidados físicos, como en los psicológicos. Los participantes consideran la vida es un nuevo nacer que está ligada a la felicidad.

E1. "Entrega y despertar del corazón de cada persona, para que nazca de nuevo y se dé cuenta de la realidad de la que está su vida en este mundo, se dé cuenta que hay una meta ultima de la vida de que no todo lo que percibimos es la realidad de todo, ya que hay mucha ilusión y a través de esa ilusión nosotros desarrollamos deseos y esos deseos cuando no están desechos generan frustración y eso nos amarga la vida".

E3. "Conciencia de Krishna a lo largo de los años con su practicante se dedica a eso 
a explicarle como servir mejor, y cual es en si la finalidad de su vida humana, para nosotros es como una gran misericordia obtener la forma de vida humana".

Respecto al cuerpo, se recogen relatos que comprenden que es una herramienta de cambios según la etapa de la vida, pero diferencian el alma como un ser eterno y verdadero para el cuidado.

E1. "El cuerpo solamente es una herramienta donde una entidad viviente "lliva" que significa alma, habita para ejecutar su "darma" es el deber de cada ser viviente, solamente es un medio con el cual no nos podemos identificar con el cuerpo... es una herramienta para ejecutar la meta final del cuerpo humano o chetra es para adorar al supremo, realizar ofrendas como por ejempló que nuestra lengua sea para hablarle a este cuerpo, es para ayudarle al dagma supremo por que el mismo krishnaa dios tiene un dagma supremo"

En este sentido, el nacer de nuevo implica una nueva oportunidad, una misericordia, un servicio. El cuerpo es una herramienta o un recipiente del alma (la entidad viviente). El alma es un ser eterno y verdadero para el cuidado.

\section{El cuidado, un estilo de vida espiritual para el cuerpo y el alma}

Entre la categoría de "cuidado", se encontró tres subcategorías que mencionaban los participantes: espiritualidad, hábitos saludables e intervenciones. El cuidado espiritual necesita ser abordado desde diferentes disciplinas que requieren de un manejo holístico e integrativo tanto desde la medicina y enfermería convencional, como desde la alternativa; lo que implica más preparación y conocimiento sobre estrategias de manejo y cuidado espiritual

E2. "somos almas espirituales, por lo mismo que te acabo de decir, no somos este cuerpo, entonces somos un alma espiritual que busca la felicidad y la verdad, eso es como nuestra naturaleza, la naturaleza de todos, todos buscamos ser felices siempre, solo que enfocamos nuestra energía en cosas que no nos van a dar la felicidad, entonces la idea es que nuestra alma encuentre la felicidad con Dios".

E3." krishna la espiritualidad es un diario devenir ósea es algo que se debe hacer todos los días como decía un devoto hace poco que escuchaba, para un devoto un día espiritual no puede comenzar sin que tienda su cama ósea yo me puse a pensar en eso hasta las personas de afuera tienden la cama, las personas que no practicas conciencia de krishna"

La espiritualidad no está mencionada como una determinada religión si no como la actitud, acción interna a la ampliación de la conciencia y un contacto continuo con los sentimientos y pensamientos superiores, también esta es mencionada como la vivencia del "amor mayor" es ese amor por la que se siente una gran plenitud y sensación de virtudes para la obtención de la razón de la existencia, como cada ser humano es único e indescriptible llevando a un resultado según su personalidad (Benito E, Barreto P, Oliver A, Galiana L, Fombuena M, Diego R., 2015).

E1. "viene de espíritu, nosotros por naturaleza estamos enlazados con una gran energía, de la cual estamos hechos se le llama sumer alma, nosotrossomos porciones de ese granespíritu...... la felicidad y espiritualidad están muy ligadas, 
espiritualidad es parte de ese principio de la gran alma, gran espíritu. Espiritualidad es muy diferente a religión, religió significa reli- de religar generar conexión con el gran espíritu, se dice que ese gran espíritu es como una fogata donde esas chispas que se desprenden somos nosotros, ya que somos independientes pero siempre tenemos que volver".

Los hábitos saludables son fundamentales para la preservación de la salud, siendo costumbres para llevar una vida sana. En los testimonios dados por los entrevistados se destaca lo esperado aunque no delimitan el probable riesgo de desnutrición por los hábitos alimenticios (Bahamondes González \& Marín Alarcón, 2015).

E1. "a través de la alimentación, donde se purifican y poco a poco se abre un portal a la espiritualidad, la revolución de la cuchara, vegetarianos, veganos".

E3. "I salud en realidad va muy de la mano con conciencia de krishna hasta el punto de que en nuestro diario vivir hay una seria cantidad de ayunos de prácticas que ayudan a limpiar el cuerpo para que uno pueda estar no solo en la parte física sino también en la parte mental que yo lo relaciono como muy directamente con parte de la salud que la mente y el cuerpo estén como muy unidos estos vedas"

El Cuidado espiritual necesita ser abordado desde diferentes disciplinas que requieren de un manejo holístico e integrativo; lo que implica más preparación y conocimiento sobre estrategias de manejo y cuidado espiritual. Se destacó el hecho del manejo del backiyoga y el Sadhana como una herramienta de relajamiento y cuidado.

E1. "Sadhana es un ritual que comprende levantarse temprano tipo 4am y empezar a cantar mantras que son muy recomendados en esta hora para hacer yoga que significa yuga o conexión con la suprema personalidad de Dios, entonces le cantamos a las 4 am estos mantras en congregación, también nosotros le bailamos y le tocamos instrumentos una hora y media antes de la salida del sol...... se abra un portal hacia los planetas espirituales y se genera una conexión donde cualquier petición que se haga en ese lapso de tiempo va a ser escuchada, cualquier cosa será atendida, es una forma de conectarse por medio de los mantras ... El Sadhana continua luego de que sale el sol con servicios se vuelve bactil o gozo en mi alma, unos estamos en restaurantes, otros en Tours donde estamos en las ecualcaldías para que hagan esa conexión con Dios, se hagan sanación, baños, además le predicamos a las demás personas razón por la cual estamos acá, predicar es despertar el alma de cada persona y así hasta la noche".

E3. "voy a dedicar un tiempito a dios me voy a sentar a escuchar una misa, voy a meditar, a hacer yoga no, para nosotros la espiritualidad es diaria"

E1. "el yoga que solo los frutos del krisma pero no quieren al Krisna, como el backiyoga, o yoga que es el adquirir los conocimientos que se adquieren con practica que se convierte en servicio a la humanidad, es volvernos consientes, es más espiritual, diferente al que se está vendiendo que es más físico".

El cuidado está relacionado con el cultivo de sí mismo, como un estilo de vida que se basa en la experiencia espiritual, que tiene como objetivo el encontrar la felicidad y la verdad. Se busca la salud de la mente y el cuerpo por eso se realizan ayunos, baños, sanaciones y se predica, para limpiar el cuerpo y cuidar el espíritu. 


\section{Las prácticas que permiten una conexión con el Krishna}

Al indagar sobre las prácticas y creencias de los participantes, describieron seis tópicos: principios, Dios, deidad, vestimenta, género y ceremonias. A continuación, se describe cada una.

E2. "El primero es: no consumir animales, no comer animales ni huevos; Ee el segundo es que no intoxicamos el cuerpo con alcohol ni drogas, porque la idea es llevar un proceso consciente ¿no? y cuando uno consume alcohol y drogas pues sus sentidos se alteran ¿no?, como que cuanta gente a cometido tantos errores por estar, en esos estados, entonces no intoxicamos el cuerpo; el tercero es no, digamos las relaciones sexuales son sagradas entonces las usamos solo para procrear dentro de un matrimonio; y el cuarto es que no jugamos juegos de azar. Entonces son como nuestros principios y bueno pues así llegué al templo".

E1 "En el caso de la intoxicación y sexo ilícito si nosotros le damos riendas sueltas a esas dos cosas nuestra energía vital se va a disminuir y genera obstáculos para tener una vida espiritual".

En la unidad de "Dios" Aleteia menciona un discurso como un ente atractivo de suprema personalidad. Los participantes destacan la importancia de tener a Dios en sus vidas

E2. Dios es "Krishna"; "Krishna" es la suprema personalidad de Dios, significa "el supremo atractivo", pero en realidad también "Krishna"....cada religión son como maneras de llegar a Dios con las que una persona se identifica ¿no?, entonces yo me identifico más con ser "hare krishna", con pertenecer a esta cultura pero otra persona puede identificarse con el catolicismo, y está bien desde que uno lo practique correctamente".

E1. "creemos en un solo dios en el señor Krisna, que significa el supremo atractivo, que es la meta más elevada de todo lo que existe por encima de los mundos materiales como estos y es una deidad a sea dios yeoba el nombre que le quieras dar es el mismo y él es el que crea sostiene y mantiene toda la creación"

E3. "Hari es el nombre de dios que traduce ladrón de corazones".

La adoración de Dios para la cultura Hare Krishna es de gran importancia porque es el acercamiento continuo a una vida espiritual.

E1. "El señor supremo entra en la Deidad realmente es una forma de adorarlo toda la mañana hay un tipo de sacerdote en el altar al cual lo despiertan pues desde la noche anterior lo dejan dormir con los gorros la pijama, así como un rey, lo levantan con un dulce, postres y un buen desayuno de cinco preparación".

E2. "Nosotros adoramos a la "Deidad" ee tres veces al día más o menos, bueno, uno de nuestros rituales por decirlo así, es cantar, muchas personas nos han visto de pronto cantar en la calle, eso se llama salir a hacer "harima", entonces como que nuestro proceso se centra mucho en el poder del canto, el poder del sonido, el sonido tiene mucha, mucha potencia... hay un estudio de un japonés que se llama "Masaru Emoto", no sé si lo has escuchado, es acerca de la importancia del sonido en el agua, ¿cómo influye? entonces ahí muestra como él, como ve los cristales del agua con diferentes palabras ¿no?, entonces cuando dice "amor" o palabras así como buenas, el agua hace unos cristales hermosos, en cambio 
cuando le manda como palabras de odio y esas cosas los cristales como que se deforman, entonces en eso también consisten ¿no? cómo purificar la mente y los sentidos y el alma por medio de cantar los nombres de dios".

E3 "es salir a cantar a las calles, es que mucha gente lo ve a uno vestido así de devoto y de una lo primero es hare hare, reaccionan a eso no porque los han visto cantar en las calles ....método de liberación es simplemente bueno suena muy simple pero en realidad es un poco complejo, recordar el nombre de dios no o cantar el nombre de dios o cantarle a la gente a cerca de dios acerca de lo que a él le gustaba hacer lo que realizaba sus glorias eso es haridan sankirtan esa es como la actividad principal que se realiza para esta era".

Otra de sus creencias es las ceremonias del fuego un medio de purificación de los momentos importantes. Lo menciona uno de los participantes.

E2. "Ceremonias de fuego son como, hay diferentes como fines para cada ceremonia, entonces digamos cuando dos personas se casan ee hacen una ceremonia de fuego, cuando las personas se inician se hace una ceremonia de fuego, son como medios purificatorios".

Las costumbres de la vestimenta a la época medieval hindú, son la que en la entrevista los participantes portaban y relataban el significado de sus prendas y joyas.

E1."La vestimenta en el caso de los hombres, puede ser negra, color azafran o fuego que significa purificador, los de blanco quiere decir que ya fueron Ramachari por un tiempo y ya se han formado y ya quieren asociarse o casarse ya están preparados espiritual y material mente para crear una grija (familia)".

E2: "aries" son blancos, significa que, blancos con una línea azul en el borde, significa que esa persona es como, esa mujer es como una monja, nosotros tenemos un tiempo en la vida, en la que tenemos que ser como "Bramacharinas" entonces eso es un proceso en el que uno no tiene novio, no está con alguien para cultivarse uno mismo, no es algo que sea de porvida, a menos de que uno quiera, sino como tú puedes ser "bramacharina" un tiempo cultivar tus estudios, tu vida espiritual y luego puedes casarte, algo así, entonces las mujeres que están vestidas de esa manera son "bramacharinas", están dedicadas a eso; lo mismo los hombres, cuando tienen un "dhoti" color azafrán como medio naranjita, es porque son "Bramacharis", entonces son monjes que están dedicados a la vida de templo... "bindi se usa como para representar el tercer ojo de la mujer y también en India lo usan para que los hombres no miren a las mujeres a los ojos, porque es un punto de distracción, miran el "bindi" y no los ojos, entonces también lo utilizan para eso".

El género es determino para caracterizar las diferencias de roles en las mujeres y hombres en la cultura hare krishna. Encontrando la importancia de los valores y principios para tratar a una mujer con la combinación de cortesía, amor y respeto.

E1 "La mujer debe ser tratada como una divinidad, es una energía bondadosa y se le daba dar protección, también a la mujer se le aconseja que no tome matrimonio con cualquier hombre, y el día a se asocien sea para que los don crezcan espiritualmente en conjunto no para que el hombre degrade a la mujer... La mujer es vista como fuente de amor 
y manutención de las entidades vivientes, ella es la energía que prácticamente nos tiene y nos da ese calor, tener la conciencia de que la mujer es como la mamá".

E2. "Las mujeres son muy respetadas ¿no? acá, haya a las mujeres se les dice madre, así tenga cinco años o veinte años o no hay tenido hijos, como que es más que los hombres tienen que ver y respetar a una mujer como si fuera su mama ¿sí?, entonces ee sí, es como para entablar ese respeto y como las mujeres son sagradas como que es, ee pues se busca mucho cuidarlas".

El rol del hombre son personas espirituales que a partir de su energía logrando obtener la auto conservación.

E1. "El hombre por lo general en los templos son monjes, han tomado bramacharia significa que han tomado un boto de celibato donde no se asocia con ninguna mujer, trata de mantener u energía plena, auto conservación, su energía sexual ya que todo esta energía se ve como conocimiento".

\section{La enfermedad y la muerte, hay un nuevo renacimiento}

En la categoría de enfermedad se encontró tres subcategorías entres estas: dolor, percepción de los cuidadores de salud y medicina ancestral. Como primera instancia el dolor es percibido para los participantes como una forma de sufrimiento siendo un experimento de la vida donde no existe la enfermedad si no la vida en el alma (16)

E3. "Para el devoto en realidad solo existe el servir entonces casi que la enfermedad no existe para un devoto porque para el devoto solo existe el servir"
E1. "El dolor es lo principal en lo que se basa este mundo natural, desde que nosotros estamos en el vientre de la madre nosotros estamos sufriendo, pero nosotros no nos acordamos, las bacterias benignas nos pican, llegan una semana donde está en posición fetal la cual uno está así recordando la posición de yoga donde estamos diciéndole a Krishna que queremos salir de acá. Al salir conocemos otro dolor el de la niñez donde nos frustramos por que no se nos cumplen los caprichos, luego adolecentes el primer amor todo lo que estamos y experimentamos en nuestra vida, cada cosa hace parte del sufrimiento" (...) El dolor es un maestro que nos dice mira por acá no es el camino el mundo material no lo es todo, entonces cuando uno empieza a madurar luego de muchos nacimientos y muertes, porque nosotros creemos en la reencarnación entonces es donde se prende esta chispa de vuelta al camino de Dios".

Desde tiempos antiguos para el manejo de las enfermedades se han utilizado los tratamientos naturales, los participantes coincidieron en que asistir a un hospital por enfermedades graves es lo correcto, son meticulosos con el consumo de químicos y medicamentos prescritos por el personal de salud.

E2 "Remedios naturales "ayurvédicos", el "ayurveda" es como la medicina de la india, pero cuando son casos ya de enfermedades, no sé, terminales o que ponen en peligro la vida claro hay que ir a un hospital".

E3. "El ayurveda es medicina en realidad entonces ayurveda es un libro demasiado gigante donde explican como un devoto un practicante de conciencia de krishnan debería alimentarse para que en el futuro en la realización de su servicio devocional no sufra pausas y sea demasiado constante". 
E1. "Los devotos deben cuidarse mutuamente, si hay alguien que se enfermó los demás lo ayudan pero no lo dejan desatendido así como una familia, así sean diferentes sin importar nacionalidad, a nivel personal claro cada uno se cuida, cada uno trata de comer a horas para no enfermarse, también recibimos información de medicina ancestral natural porque nosotros no creemos en la medicina química pues esta nos quita el dolor temporal pero el químico quedara en el cuerpo haciendo peores cosas, por esto nosotros tratamos de no consumirlos a no ser que sea ya muy grave o urgente como un operación... Es el manual de cómo debe ser tratado el cuerpo según los diferentes tipos de enfermedades y los diferentes cuerpos como gordos, flacos etc., donde la alimentación es diferente según el tipo convirtiéndose así la alimentación en su propia medicina, los médicos normales tratan con el mismo medicamento los diferentes cuerpos lo cual es malo pues el químico puede afectar a unos más que otros, o funcione más en uno que en otros, además generan más enfermedades degenerativas".

La percepción del personal de salud, los participantes fue contradictoria según vivencias de la percepción a lo que implica sobre un trato humanizado y holístico del personal.

E1. "Si yo estuviera hospitalizado seria por algo que se haya salido de control y eso les pasa mucho a los devotos todos tienen ese chip. Son influenciados por esta era una era donde hay mucha negligencia donde la juventud no toma esto enserio, cuando nosotros vemos enfermeros, médicos en los hospitales así jovencitos podemos observar que ven todo tan frio, sin escrúpulos como si los pacientes fueran pedazos de carne no hay conciencia".
E2. "Es una vocación muy admirable ¿no? como de verdad entregar su vida a cuidar a los demás eso es como servicio desinteresado en parte".

\section{La muerte como una puerta de entrada a la nueva de vida y de conexión con dios}

Finalmente se analiza la categoría de muerte, con las subcategorías reencarnación y culto en la muerte. El cuerpo continuamente tiene cambio como en las etapas de la vida y el ciclo nacimiento, enfermedad, vejez y muerte, así mismo es el alma en la muerte, pasa de un cuerpo a otro según la transición y el karma que había tenido antes de la muerte como una acción- reacción. Si se obsesiona a un mundo material y no espiritual en el momento donde el alma deja el cuerpo, regresa a pagar el karma. Bhagavad Gita indica que es posible salir de la rueda samsara o también conocida como la rueda de la reencarnación, cuando se practica el amor puro de yoga o Bhakti yoga, como lo indican los tres entrevistados.

E1. "La muerte es la puerta a volver a nacer, la muerte no existe, la muerte es algo ilusorio, solo muere el cuerpo pero tu conciencia es la que sigue viva y ella trasciende, ella está atada a tu alma o yiba. La muerte solo es el paso para la transmigración de tu alma de un cuerpo a otro, según tus deseos tu yoga...como humanos hemos pasado por diferentes vidas desde gusanos hasta perros y para haber quedado en este cuerpo humano es porque tuvimos un buen karma y Darma, este cuerpo nos permite estar más cerca de la autorrealización. Tener un cuerpo animal después de la muerte es algo muy miserable, pero gracias a la misericordia del supremo logramos tener este cuerpo humano, es por eso que debemos para la 
adoración a Dios y para hacer algo por la tierra".

E2. "Nosotroscreemosen "lavida después de la muerte" entonces por eso es importante como dedicar la vida a Dios, porque en el momento de la muerte es muy importante recordarlo, ósea a nosotros como en ese momento es lo que determina que es lo que va a pasar con nosotros después, en el justo momento de la muerte, porque dependiendo de lo que tu pienses cuando abandonas el cuerpo es como lo que va a pasar después, por decirlo de alguna manera, entonces digamos, entonces cual sería lo ideal como en el momento de la muerte recordar a Dios".

E3. "El estado de conciencia humano hemos tenido que pasar de montones, montones y montones de nacimientos como animales y árboles".

En el momento de la muerte señalan las emociones y prácticas que se convierte en un momento de transcendencia hacia la vida

E1. "Orar por el alma del difunto pues uno no sabe cómo es el karma de esa persona, nosotros oramos para que esa persona no caiga en una entidad viviente inferior o infernal, se ora para que el supremo le de otra oportunidad si aún no puede llegar a un estado espiritual....un sacerdote en la india dice que uno debe ponerle un vaso con agua y leche, pues cuando abandonamos el cuerpo y aun se identifica con su yo, su ego, entonces ella, se sentirá confundida pues se va a desesperar, entonces para calmar esta angustia uno hace unos mantras especiales y le pone su vaso con leche, así podrá aceptar el conocimiento de ya no estar en su cuerpo, ya se desprendió de lo que se identificaba y así pasa a otra etapa".
E2. "Lo ideal es cremarlo, cuando alguien, todos los devotos cuando fallecen los creman y ponen sus cenizas en un río sagrado osea los dejan fluir, porque cuando, pues nosotros creemos que cuando el cuerpo lo entierran sin cremarlo, como que el alma permanece atada al cuerpo ino? entonces la idea es como cremarlo para que el alma también pueda como irse tranquila, por decirlo de alguna manera, entonces pues el duelo, en la transición de la muerte, cuando alguien está abandonando el cuerpo, lo ideal es que los devotos estén ahí ...cuando ya abandona el cuerpo y orar mucho por él, se le ofrecen, digamos por él unos días, se le ofrecen como "remanentes de la deidad", a la "Deidad" se le ofrece como alimento, entonces uno pone una fotito de la persona y le ofrece como esos "remanentes" para que se vaya purificando en ese proceso como de cambiar de cuerpo o de cambiar de, como de estado".

E3. "El cuerpo es marcado con las 2 etílicas o 12 marcas que le hacen en el cuerpo que son como sagradas que representan que el cuerpo es un templo de dios no algunos le ponen hojas de tulacique es una plantica que tenemos aquí una foto, se la ponen en la boca y en varias partes del cuerpo como para porque bueno el tulaci es una planta sagrada se destaca a dios como un ente que determina sus vidas, que los acompaña, fortalece y consuela en los momentos difíciles".

Recopilando lo anterior, el dolor implica sufrimiento, es la base del mundo que permite reencausar el camino hacia el objetivo. Durante la enfermedad, se cuidan mutuamente y se tratan con alimentos naturales, solamente recurren al hospital ante situaciones graves de salud. Ellos evitan el uso de químicos (medicamentos químicos) porque quedan en el cuerpo. Cada cuerpo es diferente y le 
administran el mismo medicamento a una persona sin considerar las diferencias de cada cuerpo. La muerte es el camino hacia la nueva vida porque creen en la reencarnación y durante el morir es importante recordar a dios y estar en contacto con él. Después de la muerte, hay que desatar el cuerpo del alma para que esta pueda avanzar y por lo tanto, prácticas como la oración, son importantes para permitir una reencarnación en un mejor ser.

\section{DISCUSIÓN}

Los resultados anteriores, dan cuenta de la importancia que tiene el comprender la perspectiva de vida de una comunidad religiosa como los Hare Krishna en la práctica de enfermería de los cuidados paliativos. Comprender los significados de la vida, la muerte, la salud, la enfermedad, la curación, el cuerpo y alma; son necesarias para atender de manera integral las necesidades de una persona de esta cultura al final de la vida.

Teniendo en cuenta lo anterior, es necesario promover ambientes donde estas comunidades se sientan incluidas, valoradas y conservadas en la atención de salud, promoviendo el clímax espiritual que ellos buscan durante las etapas de enfermedad y de muerte.

Los participantes expresan en la entrevista sus percepciones y prácticas frente al cuidado de su propia salud, lo cual constituye una adopción de medidas en las que la alimentación tenga un sentido curativo y el uso de medicinas naturales sean aceptadas e incorporadas como parte de los tratamientos convencionales. Sus creencias llevan a un acercamiento hacia una vida armoniosa con el entorno, los seres vivientes, los animales, la naturaleza y consigo mismo.
La espiritualidad es un estilo de vida para su comunidad, cada práctica les permite conectarse con su Dios y se encuentran en una búsqueda constante de paz interior, tranquilidad y felicidad. Incluso en la muerte, la necesidad de esa conexión se hace mayor y en este sentido, prácticas como la oración de seres queridos, amigos y miembros de la comunidad debe respetarse en los espacios de atención clínica o en los hogares donde se esté muriendo. La práctica de una despedida con este mundo y de desprendimiento del cuerpo, es algo aceptado para ellos que puede promoverse como parte de las intervenciones espirituales de enfermería al final de la vida.

Así mismo, el buen morir está relacionado con la espiritualidad, donde la muerte es algo natural, un nuevo paso a la nueva vida donde el cuerpo es el que tiene un final, pero el alma se reencarna según el Karma de cada ser.

Por otra parte, es importante considerar que prácticas que son llevadas a cabo por los Hare Krisnha, manifestadas por las personas entrevistadas (como el yoga, la meditación, la alimentación vegetariana o el canto), se convierten en un aspecto muy importante a la hora de brindar un cuidado de enfermería en el ámbito hospitalario, debido a las limitaciones propias del ambiente clínico, que vulneran de alguna forma, el desarrollo normal de sus prácticamente.

\section{CONCLUSIÓN}

La cultura religiosa para los Hare Krishna influye en gran medida en la salud y percepción de la enfermedad. Es importante que el equipo de salud y de enfermería se preparen para brindar cuidados que sean culturalmente competentes y que tengan en cuenta las 
particularidades de los sujetos de cuidado, especialmente en periodos tan importantes como puede ser el proceso de muerte. Los ambientes y las prácticas de cuidados paliativos, deben considerar elementos de la cosmovisión de las personas y en este sentido deben adaptarse al cuidado de cada persona.

Existe poca literatura en el país que relaciona la perspectiva de la comunidad Krishna desde la salud y ninguna existente sobre enfermería, por cual es importante profundizar en futuros estudios frente a esta temática.

\section{AGRADECIMIENTOS}

Docente de la Maestría en Enfermería en cuidados Paliativos Doctora Lina María Vargas Escobar por su orientación y dedicación.

\section{REFERENCIAS BIBLIOGRAFICAS}

1. Arteaga, J., \& Ibáñez, A. M. (2018, enero). Migración en la ELCA: ¿Quiénes migran, por qué motivos y cuáles son los beneficios potenciales? Recuperado 1 de octubre de 2019, de Económia Uniandes website: https://economia.uniandes.edu.co/component/ booklibrary/478/view/46/Documentos\%20 CEDE/1107/migracion-en-la-elca-quienes-migranpor-que-motivos-y-cuales-son-los-beneficiospotenciales

2. Bahamondes González, L., \& Marín Alarcón, N. (2015). Expansión Hare Krishna en contextos de crisis: Resignificando la movilización social desde una perspectiva religiosa en Chile. https://doi.org/ DOI: 10.3989/rdtp.2015.02.004

3. Barreto, P., Fombuena, M., Diego, R., Galiana, L., Oliver, A., \& Benito, E. (2015). Bienestar emocional y espiritualidad al final de la vida. Medicina Paliativa, 22(1), 25-32. https://doi.org/10.1016/j. medipa.2013.02.002

4. Bhaktivedanta S, A. C. (2009). Bhaktipedia-El Desafío Hare Krishna. Recuperado 1 de octubre de 2019, de http://bhaktipedia.org/espanol/index.phpn=bhaktivedanta_swami.desafio_hare_krsna.html

5. Carrasquilla Baza, D., \& Pérez Quintero, C. (2018). Experiencias del cuidado de enfermería en contexto intercultural. Cultura de los Cuidados Revista de Enfermería y Humanidades, (51). https://doi. org/10.14198/cuid.2018.51.14

6. Congreso de la República. Ley 1438 de 2011. , Pub. L. No. 1438 (2015).

7. Congreso de la República. Ley 1751 de 2015. , Pub. L. No. Ley 1751 de 2015 (2015).

8. Const. (1991). Constitución política de Colombia de 1991. Bogotá: Cooperativa Editorial Magisterio.

9. Guirao-Goris, J. A. (2013). La espiritualidad como dimensión de la concepción holística de salud. Revista ENE de Enfermería, 7(1). Recuperado de http://eneenfermeria.org/ojs/index.php/ENE/article/view/252

10. Hernández Sampieri, R., Fernández Collado, C., \& Baptista Lucio, P. (2014). Metodología de la investigación. México, D.F.: McGraw-Hill Education.

11. ISKON. (2019). Asociación Internacional para la Conciencia de Krishna. En Wikipedia, la enciclopedia libre. Recuperado de https://es.wikipedia.org/w/ index.php?title=Asociaci\%C3\%B3n_Internacional_ para_la_Conciencia_de_Krishna\&oldid=119049668

12. Ketola, K. (2004). The Hare Krishna and the Counterculture in the Light of the Theory of Divergent Modes of Religiosity. Method \&amp; Theory in the Study of Religion, 16(3), 301-320. https://doi.org/10.1163/1570068042652301

13. McFarland, M. R., Mixer, S. J., Webhe-Alamah, H., \& Burk, R. (2012). Ethnonursing: A Qualitative Research Method for Studying Culturally Competent Care across Disciplines. International Journal of Qualitative Methods, 11(3), 259-279. https://doi. org/10.1177/160940691201100306

14. Pineda, E. B., Alvarado, E. L. de, \& Hernández de Canales, F. (1994). Metodología de la investigación: Manual para el desarrollo de personal de salud. Washington, D.C.: Organización Panamericana de la Salud: Organización Mundial de la Salud.

15. World Health Organization. (2019). WHO | WHO Definition of Palliative Care. Recuperado 1 de octubre de 2019, de WHO website: https://www. who.int/cancer/palliative/definition/en/ 


\section{Imagen No.1}
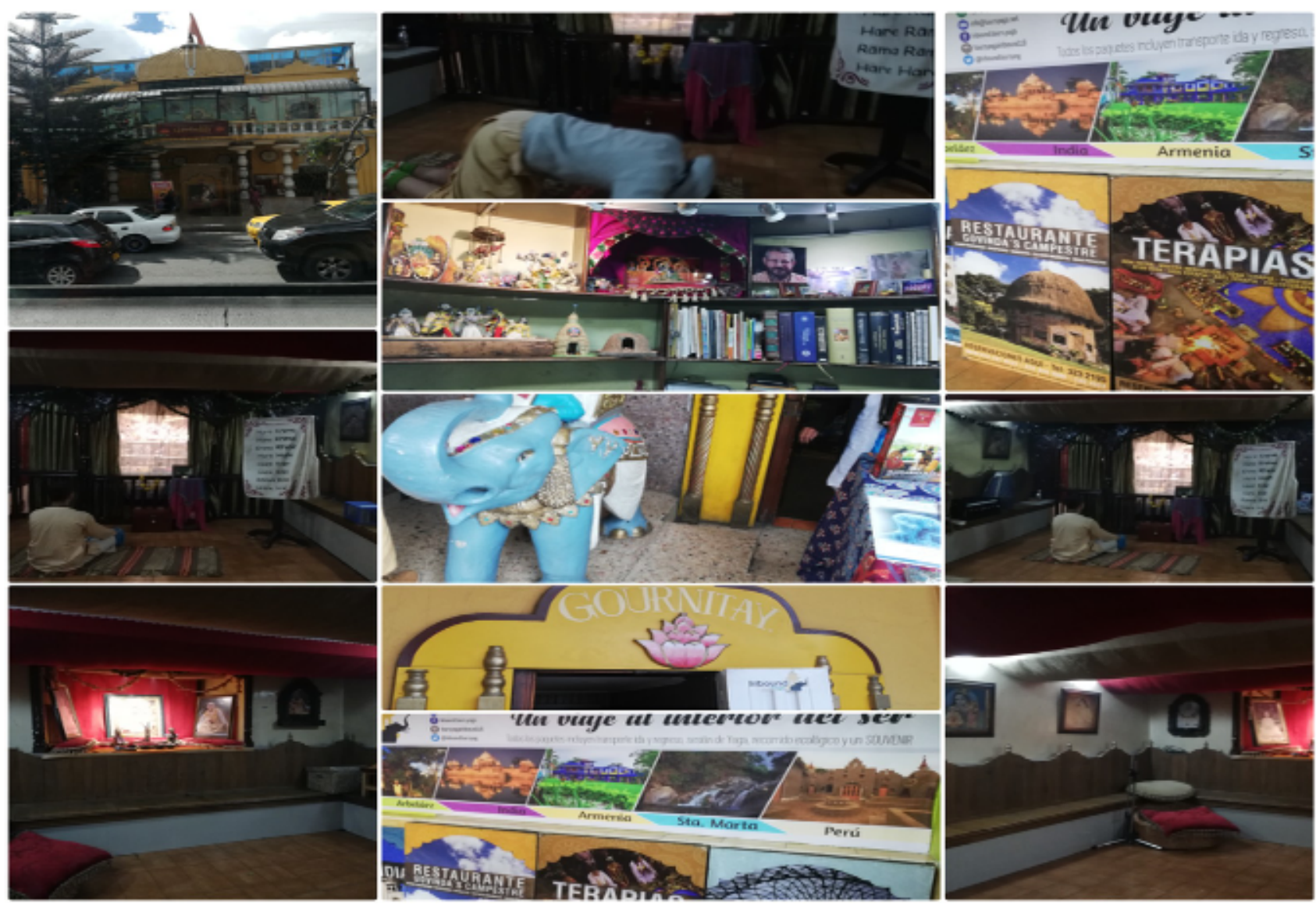

Carreño, S. (2018). Hare Krishna. [Imagen propia]. 